\title{
Stress-dependent elastic properties of shales: Measurement and modeling
}

\author{
Marina Pervukhina and Dave Dewhurst, CSIRO Petroleum, Perth, Australia \\ BorIS GUREVICH, Curtin University of Technology, Perth, Australia \\ UtPALENDU KUILA, Colorado School of Mines, Golden, USA \\ TONy SIGGINS, CSIRO Petroleum, Melbourne, Australia \\ MARK RAVEN, CSIRO Land and Water, Adelaide, Australia \\ HeGE M. NoRDGARD BolAs, StatoilHydro Research Centre, Trondheim, Norway
}

D elastic properties of shales is insufficient as it is based on a limited number of observations caused by the time-consuming nature of testing resulting from their low permeability. Though it is well known that shales are highly anisotropic and assumed to be transversely isotropic (TI) media, few laboratory experiments have been carried out for measuring the five elastic constants that define TI media on well-preserved shales. Many previous measurements were made without control of pore pressure, which is crucial for the determination of shale elastic properties.

Modeling shale elastic properties is difficult because of the complex composite nature of shales and the lack of data for anisotropic elastic properties of individual clay minerals. Values of elastic parameters of clay minerals reported in the literature vary substantially between different publications (Katahara, 1996; Vanorio et al., 2003; Bayuk et al., 2007). It is becoming increasingly clear that anisotropy parameters of shales are defined at least as much by the properties of contacts between clay particles as by the intrinsic properties of the clay minerals (Sayers, 1999). A more radical idea was recently proposed by Ulm and Abousleiman (2006) who suggested that the properties of the wet clay pack (without the silt fraction) are entirely defined by the claypacking density and independent of clay mineralogy. This concept (which we call clay-packing density or CPD) was confirmed by Ortega et al. (2007) using the nano-indentation measurements on a number of shales.

In this study, we report measurements of the five elastic constants for four sets of shales (although only two sets are fully detailed here) under mean effective stresses between 10 and $90 \mathrm{MPa}$ with controlled pore pressure. The elastic constants of these shales are then used to test the CPD concept by extracting the properties of clay packs and plotting them against the packing density. The results of this analysis show trends consistent with CPD. At the same time, the elastic properties of the wet clay packs show noticeable variations with effective pressure, while clay-packing density remains almost unchanged. This suggests that the pressure variation of elastic constants is caused by the closure of "soft porosity," that is, pores that form a tiny fraction of total pore space but have a significant effect on elastic properties because of their small aspect ratio. We attempt to quantify this effect using an anisotropic soft porosity model of Shapiro and Kaselow (2005). Our data form a good fit to this model.

Samples and methodology. Four suites of shale results are compared with models in this paper, and two sets of detailed experimental results are shown. The shales are from the North Sea, Officer Basin, Bass Basin, and Carnarvon Basin. The shales have diverse compositions and physical properties. Details can be found in Dewhurst and Siggins (2006) and Dewhurst et al. (2007). The diversity of these shales

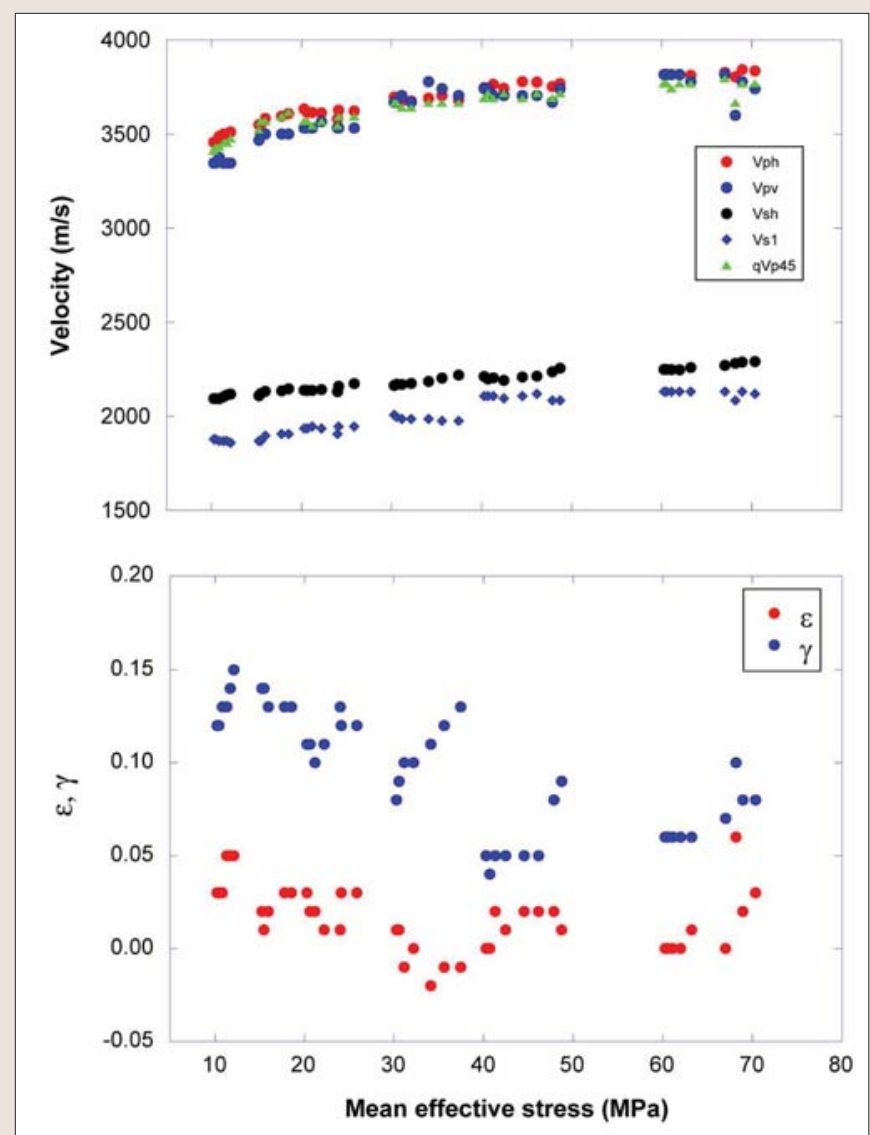

Figure 1. (top) Velocity mean effective stress plot for the North Sea shale. (bottom) P-wave anisotropy $(\varepsilon)$ is scattered but relatively unchanged during individual loading stages with increasing mean effective stress, while S-wave anisotropy $(\gamma)$ decreases with confining pressure but increases during each loading increment.

allows sample characterization in terms of silt fraction and clay-packing density. Silt fractions lie between 25 and $65 \%$, while clay-packing density is in the range of $\sim 70-90 \%$. Considerable variability of both properties is observed between the shales from different basins and also between the samples of shale from the same basin, especially for the Officer Basin shales.

Consolidated, undrained multistage triaxial tests were performed on shale core plugs at successively greater confining pressures to determine a failure envelope with a pore pressure of $5 \mathrm{MPa}$. Samples were loaded axially in an undrained state at a low-strain rate, while stress, strain, and pore-pressure changes were monitored. Ultrasonic measurements were made along these stress-strain curves from the isotropic stress condition to the maximum differential stress during each stage. We used an experimental configuration that allows the full elastic tensor to be calculated 


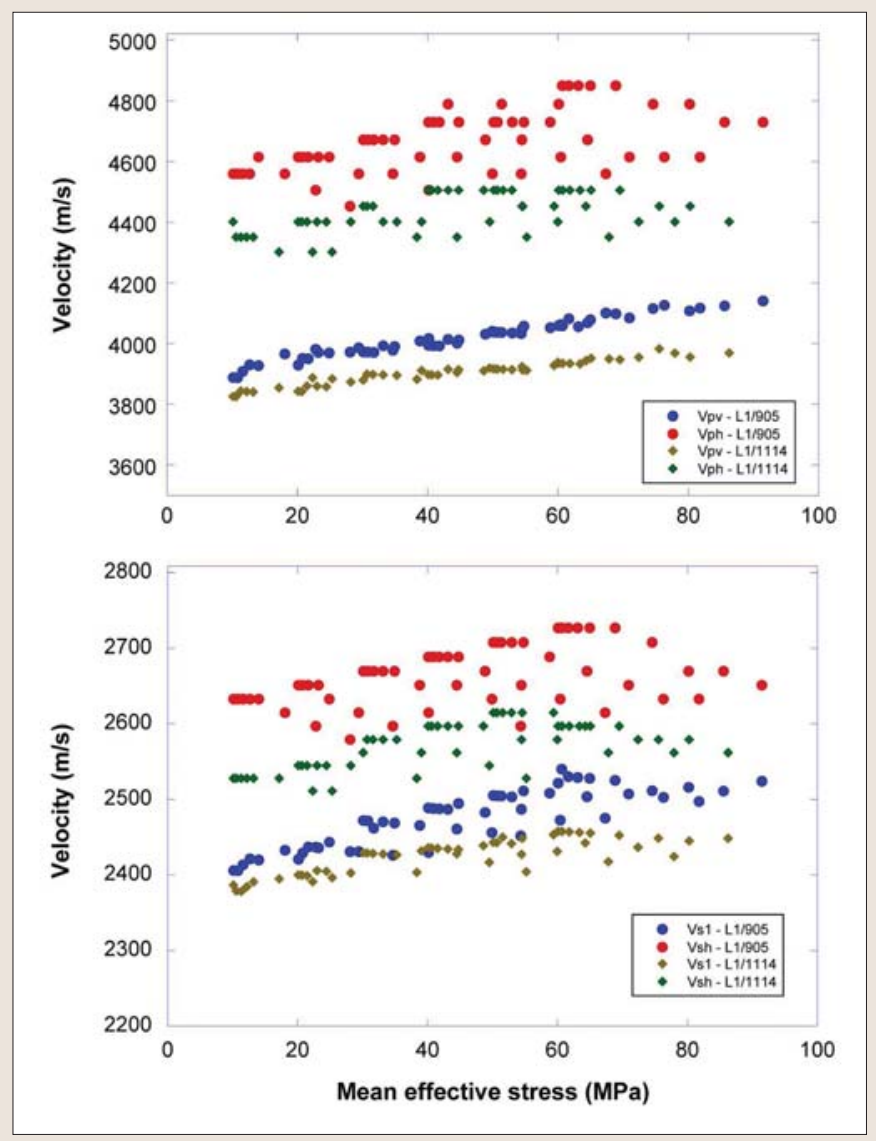

Figure 2. P-wave (top) and S-wave (bottom) velocities plotted as a function of mean effective stress in Officer Basin shales. All velocities increase in a linear trend with increasing effective stress. Note that $V_{p h}$ and $V_{\text {sh }}$ are more scattered than the vertical velocities.

from a single core, assuming that the shale is a TI medium (Dewhurst and Siggins, 2006).

Experimental results. Rock physics measurements were made during the various deformation stages on a horizontal core plug of North Sea shale. $V_{\mathrm{pv}}$ increases from $\sim 3300$ $\mathrm{m} / \mathrm{s}$ to $\sim 3800 \mathrm{~m} / \mathrm{s}$ over the mean effective stress range measured of 10-70 MPa. It is also noticeable that within most of the individual axial stress increments, $\mathrm{V}_{\mathrm{pv}}$ either remains constant or falls as differential stress increases. $V_{\text {ph }}$ is generally slightly higher than $\mathrm{V}_{\mathrm{pv}}$ (Figure 1 ), indicating slight $\mathrm{P}$-wave anisotropy, and, in general, within the individual axial loading stages, $\mathrm{V}_{\mathrm{ph}}$ either remains almost constant or increases. As there seemingly is little P-wave anisotropy, it is not surprising that $\mathrm{qV}_{\mathrm{p} 45}$ is of a similar magnitude to $\mathrm{V}_{\mathrm{ph}}$ and $\mathrm{V}_{\mathrm{pv}}$. S-wave velocity, however, shows more anisotropy than P-wave velocity in this North Sea shale (Figure 1). $\mathrm{V}_{\mathrm{s} 1}$ increases from $\sim 1800 \mathrm{~m} / \mathrm{s}$ to $\sim 2100 \mathrm{~m} / \mathrm{s}$ over the stress range investigated, although it decreases with increasing mean effective (and therefore differential) stress during each individual loading increment. $\mathrm{V}_{\mathrm{sh}}$ increases from 2100$\sim 2300 \mathrm{~m} / \mathrm{s}$ as mean effective stress increases to $70 \mathrm{MPa}$, but within each loading increment, velocity generally increases, if slightly.

Thomsen's anisotropy parameters are also shown in Figure 1, with $\varepsilon$ being slightly scattered, although this is likely caused by its proximity to zero (i.e., almost isotropic). However, $\gamma$ is moderately high $(\sim 10 \%)$ and generally decreases as confining pressure increases. It flattens off at mean effective stresses higher than $\sim 40 \mathrm{MPa}$, but increases during each individual loading cycle.
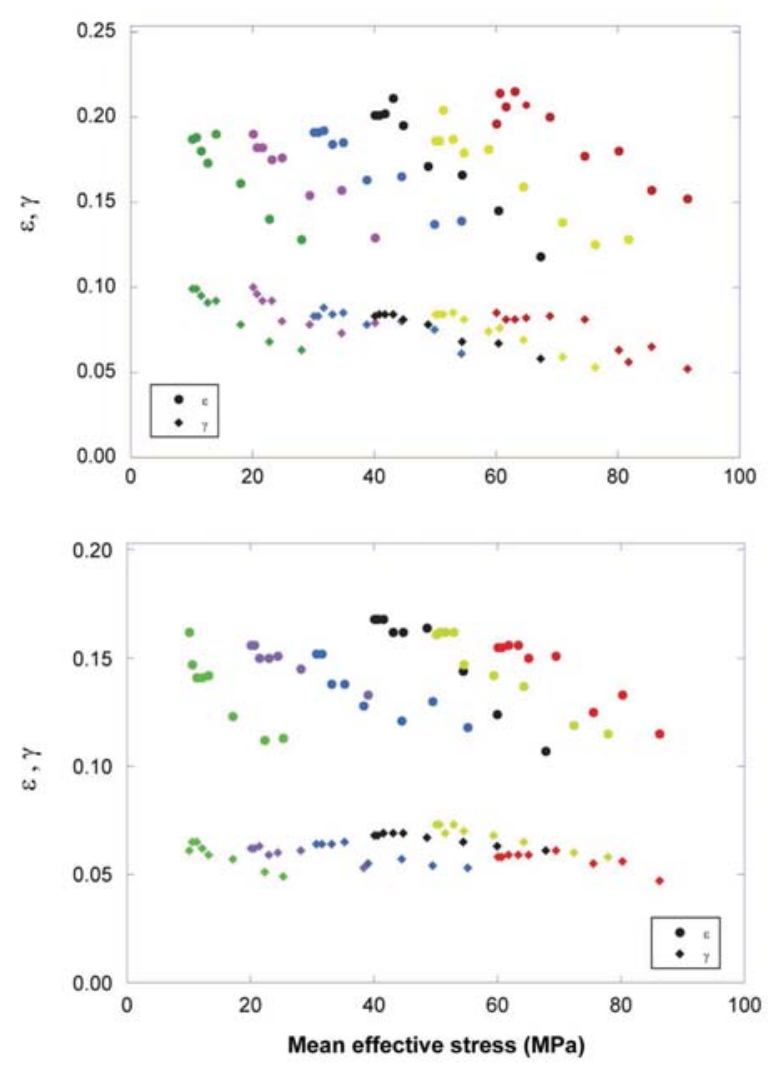

Figure 3. Influence of mean effective stress on $\varepsilon$ and $\gamma$ for Officer Basin shales. Different colors represent different confining pressure stages. Note that anisotropy is almost independent of confining pressure but $\varepsilon$, especially, decreases with increasing differential stress.

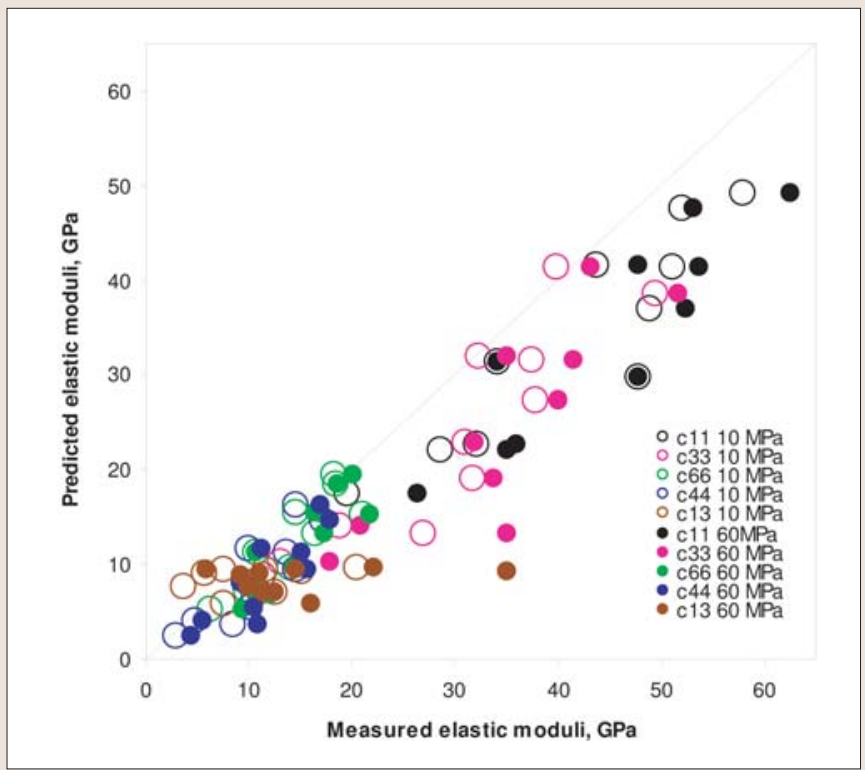

Figure 4. Comparison of predicted and experimentally measured elastic constants of shales at ultrasonic frequencies. Elastic constants $c_{11}, c_{33}, c_{66}$, $c_{44}$, and $c_{13}$ are shown by different colors. The open and solid symbols show the constants measured at mean effective stress of $10 \mathrm{MPa}$ and 60 $\mathrm{MPa}$, respectively.

$V_{P}$ and $V_{S}$ were measured for two Officer Basin shale samples (L1/905 and L1/1114) plugged normal to bedding. The results of the velocity measurements on these two samples are shown as a function of effective stress in Figure 2. 

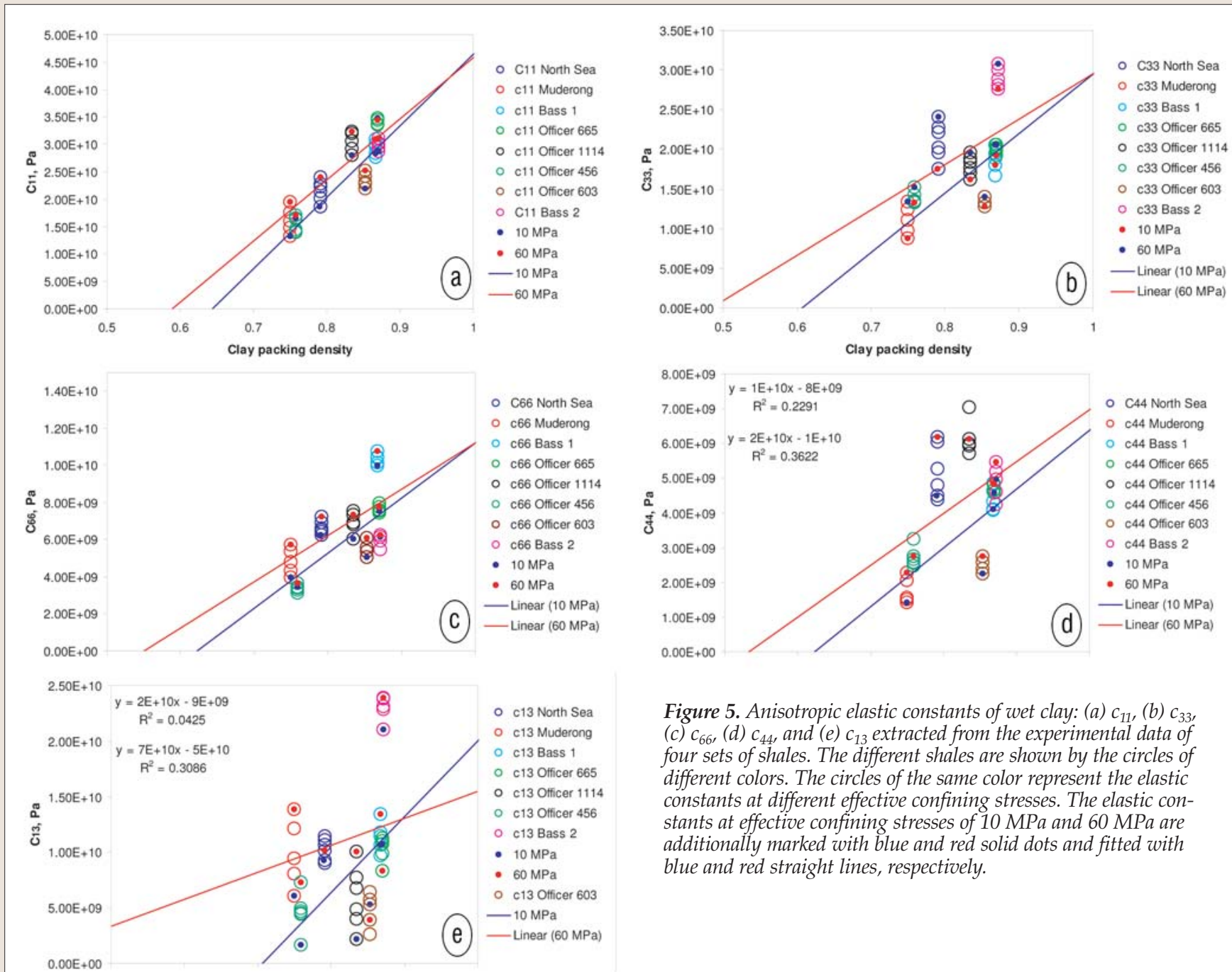

Figure 5. Anisotropic elastic constants of wet clay: (a) $c_{11}$, (b) $c_{33}$, (c) $c_{66},(d) c_{44}$, and (e) $c_{13}$ extracted from the experimental data of four sets of shales. The different shales are shown by the circles of different colors. The circles of the same color represent the elastic constants at different effective confining stresses. The elastic constants at effective confining stresses of $10 \mathrm{MPa}$ and $60 \mathrm{MPa}$ are additionally marked with blue and red solid dots and fitted with blue and red straight lines, respectively.

The velocities in L1/905 are greater than that of sample L1/1114 because of its lower porosity, but both samples show similar trends. With increasing mean effective stress, $\mathrm{V}_{\mathrm{pv}}$ and $\mathrm{V}_{\mathrm{s} 1}$ in both samples generally rise in an almost linear fashion. $V_{\mathrm{ph}}$ and $\mathrm{V}_{\mathrm{sh}}$ in both samples show more scatter than their vertical counterparts and appear to decrease within individual loading cycles.

These shales are moderately anisotropic, and the anisotropy parameters are sensitive to differential stress change. The effects of different levels of confining pressure and differential stress on the ultrasonic anisotropy are shown by color-coding the plots in Figure 3. Each color represents a particular confining pressure level. It is evident that both $\varepsilon$ and $\gamma$ are unaffected by confining pressure in these particular shales, but during each cycle of axial loading, they decrease with increasing differential stress.

Modeling shale elastic properties. Modeling elastic properties of shales is extremely challenging because of uncertain properties of clay minerals, bound water, and the nature of contacts between clay particles. However, Ulm and Abousleiman (2006) suggested that all this complexity can be expressed through a single parameter, clay-packing density, defined as $\eta=1-\phi_{c}$, where $\phi_{c}$ is the porosity of the wet clay pack. According to this concept, $\eta$ uniquely defines the properties of wet clay packs (and hence shales) through a simple linear relationship $c_{i j}=C(\eta-0.5)$, where $C_{i j}$ is a unique set of elastic constants at $\eta=1$ that are independent of clay mineralogy. Shale is then modeled as a mixture of anisotropic wet clay and isotropic silt grains using an effective medium theory. This clay-packing density concept was recently tested by Ortega et al. (2007) using nano-indentation measurements on eight different shales. By inverting shale properties using the anisotropic self-consistent approximation (SCA; e.g., Hornby et al., 1994), they obtained the following unique set of elastic constants of clay at $\eta=1$ : $C_{11}=44.9$ $\mathrm{GPa}, C_{33}=24.2 \mathrm{GPa}, C_{13}=18.1 \mathrm{GPa}, C_{44}=3.7 \mathrm{GPa}$, and $C_{66}=11.6$ $\mathrm{GPa}$.

In order to test the applicability of CPD model, we calculate elastic constants for four sets of shales using the SCA, incorporating the elastic constants $C_{i j}$ of clay at $\eta=1$ suggested by Ortega et al., and assuming that they can be rescaled to different clay-packing densities as $c_{i j}=2 C_{i j}(\eta-0.5)$. The silt grains were assumed to be spherical and to consist of quartz with $K=36 \mathrm{GPa}$ and $\mu=45 \mathrm{GPa}$.

The predicted shale-elastic constants are shown in Figure 4 along with the experimentally measured constants at ultrasonic frequencies. Measurements at effective confining stresses of $10 \mathrm{MPa}$ and $60 \mathrm{MPa}$ are shown by open and solid circles, respectively. Predicted moduli at the stresses of 10 $\mathrm{MPa}$ and $60 \mathrm{MPa}$ are the same since the porosity of shales changes by less than $1 \%$ during deformation, while the measured elastic constants change by up to $20 \%$ (10 GPa). The predicted elastic constants show a similar trend as the mea- 
sured constants but with a systematic deviation of about $13 \%$. This suggests that the CPD model does not account for the variability of shale properties in our experiments.

In order to further explore the properties of clays, we developed an "inverse procedure" to estimate claymatrix elastic constants from measured elastic properties of shale by extraction of silt material. To do this, we use the anisotropic differential effective media (DEM) approach (e.g., Nishizawa, 1982) to obtain elastic constants of clays. In our view, DEM is a

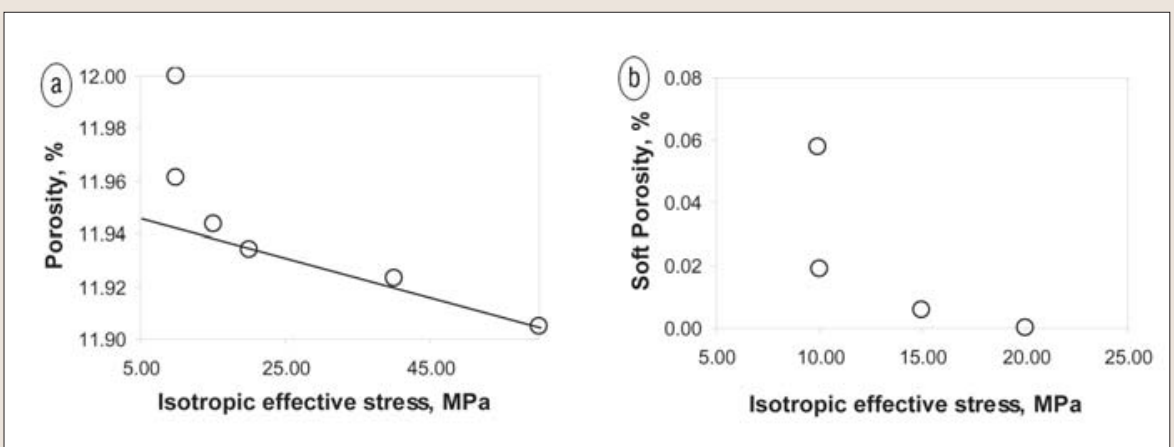

Figure 6. Porosity versus isotropic effective stress for an Officer Basin shale: (a) total porosity and (b) soft porosity. more appropriate method than SCA in

the case of shales where silt is generally distributed as isolated inclusions floating in load-bearing clay.

Figure 5 shows clay-elastic constants versus clay-packing density. The different shales are shown in Figure 5 by the circles of different colors and the circles of the same color represent the elastic constants at different effective confining stresses. The elastic constants at effective confining stresses of $10 \mathrm{MPa}$ and $60 \mathrm{MPa}$ are additionally marked with blue and red solid dots and fitted with blue and red straight lines, respectively. Despite the large dispersion, the clay-elastic constants demonstrate an obvious increase with increase of clay-packing density. The difference between the linear trends decreases with the increased clay-packing density and almost vanishes at $\eta=1$. The pressure-average of the limits of the elastic constants at $\eta=1$ are $C_{11}=46.4 \mathrm{GPa}, C_{33}=29.9 \mathrm{GPa}$, $C_{13}=17.9 \mathrm{GPa}, C_{44}=6.7 \mathrm{GPa}$, and $C_{66}=11.2 \mathrm{GPa}$. These elastic constants are similar to, but somewhat higher than, those obtained by Ortega et al. (2007).

Our observations show that the CPD model does a reasonably good job at predicting the overall behavior of elastic moduli of wet shales, but does not account for the effect of pressure. In the next section, we attempt to account for this dependency using the concept of soft porosity.

Stress dependency of elastic constants. The fact that the elastic properties of shales show much stronger variation with pressure than the total porosity suggests a causal relationship with the closure of highly compliant pores. These pores may occupy a very small fraction of the overall pore space, but have a disproportionate effect on elastic properties of rocks caused by their very small aspect ratio (Shapiro, 2003; Mavko and Jizba, 1991). In the following section, we investigate the presence of soft porosity in shales and its effect on the variation of shale-elastic constants with isotropic effective stress. We calculate the variation of the total porosity using the measured change of the length of the sample along the axis of symmetry (Figure 6a). The soft porosity is estimated from the total porosity using the recipe of Mavko and Jizba (1991), namely, stiff porosity is supposed to change linearly with the effective stress, and the soft porosity is assumed to be closed at the maximal effective stress (Figure 6). We subtracted the linear trend from the total porosity to calculate soft-porosity values at different values of isotropic effective stresses. The obtained soft porosity is presented in Figure $6 \mathrm{~b}$ and is of the same order of magnitude, but somewhat lower, than was reported for sandstones by Mavko and Jizba.

The effect of the closure of soft porosity at the isotropic effective stress of $60 \mathrm{MPa}$ on the relative variation of elastic constants $\left(c_{i j}^{60}-c_{i j}^{10}\right) / c_{i j}^{60}$ is shown in Figure 7 , where $c_{i j}^{60}$ and $c_{i j}^{10}$ are elastic constants under the isotropic effective stress

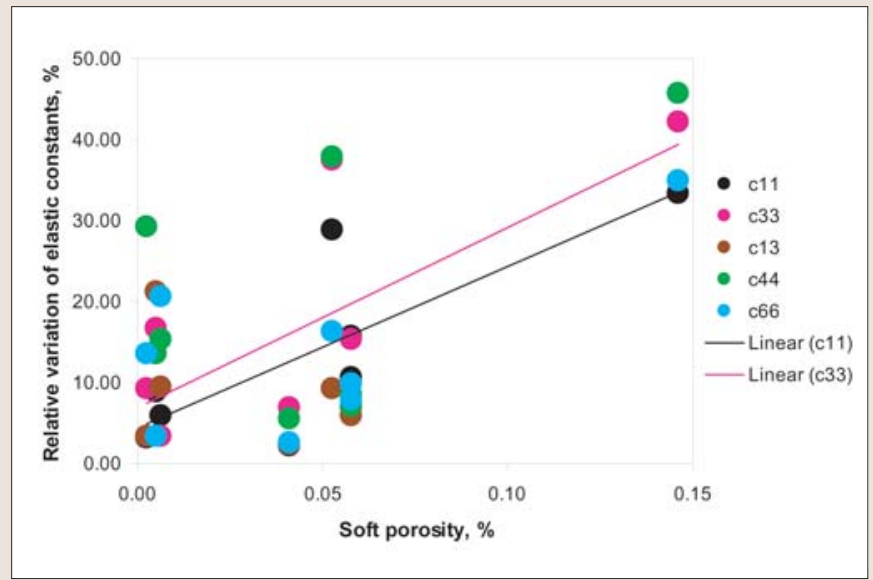

Figure 7. Correlation between soft porosity and relative variations of elastic constants with stress, $\left(c_{i j}^{60}-c_{i j}^{10}\right) / c_{i j}^{00}$, where $c_{i j}^{10}$ and $c_{i j}^{60}$ are elastic constants under the isotropic effective stress of $10 \mathrm{MPa}$ and $60 \mathrm{MPa}$, respectively.

of $10 \mathrm{MPa}$ and $60 \mathrm{MPa}$, respectively. The higher the value of the soft porosity observed in the shale, the larger the relative variation of elastic constants with increase of isotropic effective stress from $10 \mathrm{MPa}$ to $60 \mathrm{MPa}$. The variations of the clay-elastic constants demonstrate reasonable correlation with soft porosity, confirming that the stress dependency of elastic constants is caused by the closing of soft porosity.

A model of the effect of soft porosity on stress dependency of elastic properties of anisotropic rocks was recently proposed by Shapiro and Kaselow (2005). For the case of TI media and isotropic stress $P$, the stress dependencies of the compliances $S_{i i}$ can be written as follows:

$$
\begin{gathered}
S_{11}=S_{22}=S_{11}^{s}+k_{1} \phi_{1}^{c 0} e^{-k_{1} P} \\
S_{33}=S_{33}^{s}+k_{3} \phi_{3}^{c 0} e^{-k_{3} P} \\
S_{44}=S_{55}=S_{44}^{s}+\left(k_{2} \phi_{2}^{c 0}+k_{4} \phi_{1}^{c 0}\right) e^{-k_{1}^{P}}+k_{5} \phi_{3}^{c 0} e^{-k_{3} P} \\
S_{66}=S_{66}^{s}+2 k_{1} \phi_{1}^{c 0} e^{-k_{1} P}
\end{gathered}
$$

Here $S_{i i}^{s}$ are compliances of samples with the closed soft porosity, $\phi_{i}^{c 0}$ is so-called generalized soft porosity that is equal to soft porosity multiplied by volume-averaged hypothetic strain that results from the closure of the compliant part of the pore space in the $i$-direction, and $k_{i}$ are coeffi- 


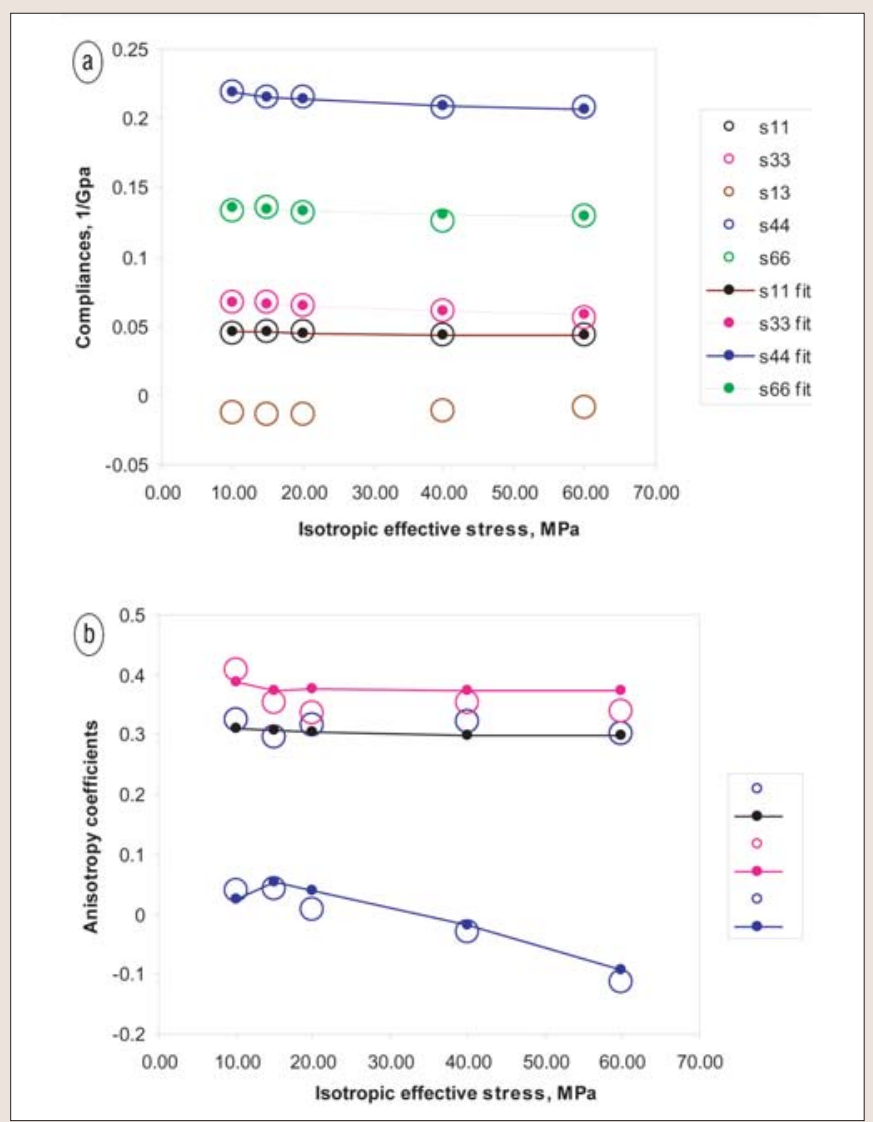

Figure 8. Measured and fitted elastic properties of clay for an Officer Basin shale: (a) compliances and (b) Thomsen's anisotropy parameters.

cients that are specific for a particular clay and depend on stress sensitivities of the clay and on elastic moduli of the hypothetic body with closed-compliant porosity. Shapiro and Kaselow derived Equations 1-4 for dry (or drained) moduli and suggested using anisotropic Gassmann (or BrownKorringa) equations to obtain saturated moduli. However, anisotropic Gassmann theory is not applicable to shales at ultrasonic frequencies, as fluid pressure does not have time to equilibrate within the wave period. Instead, Ciz and Shapiro (2007) suggest that Equations 1-4 can be applied directly to the saturated moduli of shales and show good consistency with ultrasonic measurements on a Jurassic North Sea shale. We use the same approach here.

We approximated the measured compliances with Equations 1-4 using the Levenberg-Marquardt algorithm to minimize the sum of the squares of the nonlinear functions. The results of fitting the compliances of an Officer Basin shale are shown in Figure 8a. The fitted compliances are then used to calculate Thomsen's anisotropy parameters. The measured and calculated anisotropy parameters are shown in Figure $8 \mathrm{~b}$. Both the obtained compliances and the anisotropy parameters are in good agreement with the measured ones.

The element $\phi_{1}^{c 0}$ of the tensor of the generalized compliant porosity can be estimated from the fitting coefficients of Equation 1 as a ratio of the fitting coefficient in front of the exponential to the coefficient $k_{1}$ in the exponent. In other words, $\phi_{1}^{c 0}$ is the total soft porosity multiplied by the volume-averaged axial strain. Note that the porosity shown in Figure 6 is estimated from the variation of the cylinder length along the same axis; hence, the two estimates are expected to be consistent. The compliant porosity predicted from the fitting coefficients of Equation 1 is shown in Figure

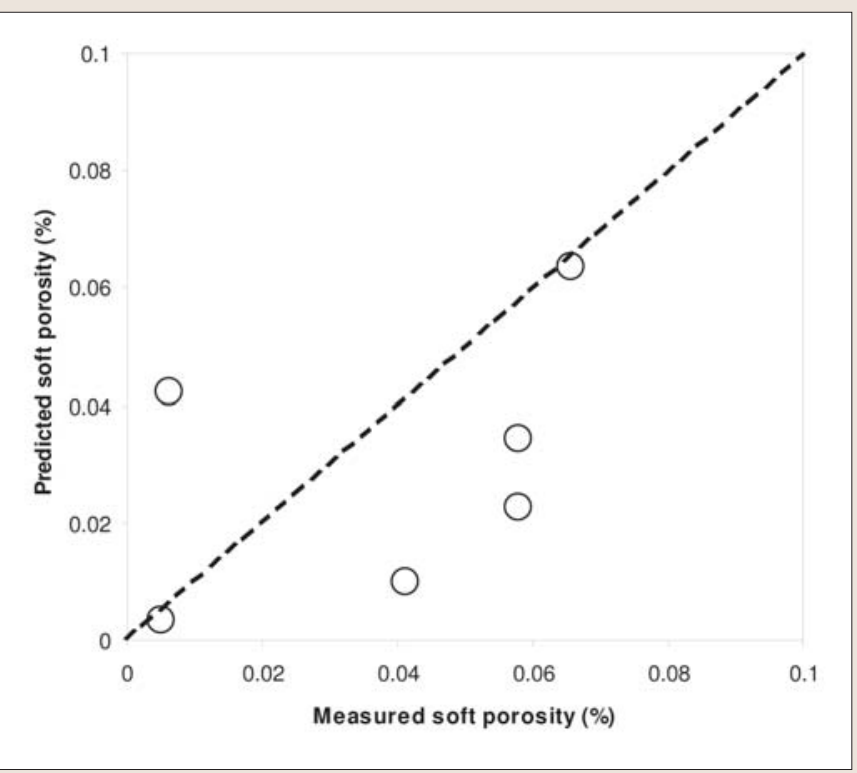

Figure 9. Measured soft porosity versus soft porosity predicted using the fitting coefficients in Equation 1.

9 in comparison with the soft porosity estimated from the experimentally measured variations of the total porosity (using the method of Figure 6). Despite a significant scatter, obtained soft porosity shows clear correlation with estimates obtained from the measured total porosity. This shows that the soft porosity can be estimated from the fitting coefficients of Equations 1-4.

Discussion and conclusions. In the North Sea shale, S-wave anisotropy increases within each individual loading stage as differential stress increases. As confining pressure increases from stage to stage, S-wave anisotropy decreases at any given differential pressure up to mean effective stresses of about $40 \mathrm{MPa}$ and then becomes approximately constant. Therefore, it would appear that application of an isotropic stress field (i.e., confining pressure) serves to close pre-existing cracks in the shale, reducing S-wave anisotropy to that resulting from fabric alignment alone at mean effective stresses above $40 \mathrm{MPa}$. However, increasing the differential stress causes anisotropy to increase through opening and propagation of bedding-parallel fractures that are exactly aligned with the maximum stress direction.

In the case of the Officer Basin shales (Figures 2 and 3), the maximum principal stress is acting perpendicular to bedding and has different effects on vertical and horizontal velocities. Microcracks present inherently in shales are generally bedding parallel and in these tests are oriented normal to bedding and are likely to close with increasing load. This can account for the decreasing anisotropy with increasing differential stress. However, continued deformation and increased stress anisotropy tends to initiate fracture planes perpendicular to the minimum stress, therefore reducing $V_{\mathrm{ph}}$ and $V_{\mathrm{sh}}$.

The contrast between the anisotropy results on core plugs deformed normal and parallel to bedding thus emphasize the importance of fabric and structural anisotropies relative to the prevailing stress field for wave propagation in inherently anisotropic media such as shales.

The measurements were used to test the model developed by Ortega et al. (2007) that calculates shale elastic constants on the basis of silt fraction, clay-packing density, and a set of elastic constants derived on the basis of the nano- 
indentation technique. The model performs reasonably well on our data, but gives systematically lower values of elastic constants. This may indicate that the direct effect of shale mineralogy, ignored in this model, is significant. Alternatively, this may be explained by the dispersion between the frequencies of nano-indentation and ultrasonic measurements.

The measurements also show a noticeable variation of elastic properties of shales (and wet clays) with pressure. At the same time, variation of the total porosity with pressure is minimal (much less than 1\% absolute porosity). Therefore, the stress dependency of shale (or clay) properties cannot be explained by the change of total porosity (or clay-packing density). In view of this, we have attempted to model the pressure dependency of shale properties by using the concept of soft porosity. Specifically, we extracted the soft porosity in two ways: (1) from the measured pressure variation of total porosity, and (2) from fitting coefficients of the anisotropic stress-sensitivity model of Shapiro and Kaselow. The two estimates of soft porosity show the same order of magnitude, confirming the validity of the soft porosity concept.

Suggested reading. "Elastic moduli of anisotropic clay" by Bayuk et al. (GEOPHYSICS, 2007). "Stress-dependent anisotropy in transversely isotropic rocks: Comparison between theory and laboratory experiment on shale" by Ciz and Shapiro (submitted to GEOPHYSICS). "Impact of fabric, microcracks, and stress field on shale anisotropy" by Dewhurst and Siggins (Geophysical Journal International, 2006). "Elastic and Petrophysical Properties of Shales" by Dewhurst et al. (presented at the ASEG 2007 con- ference). "Anisotropic effective-medium modeling of the elastic properties of shales" by Hornby et al. (GEOPHYSICS, 1994). "Clay-mineral elastic properties" by Katahara (SEG 1996 Expanded Abstracts). "Estimated grain-scale fluid effects on velocity dispersion in rocks" by Mavko and Jizba (GEOPHYSICS, 1991). "Seismic velocity anisotropy in a medium containing oriented cracks-Transversely isotropic case" by Nishizawa (Journal of Physics of the Earth,1982). "The effect of the nanogranular nature of shale on their poroelastic behavior" by Ortega et al. (Acta Geotechnica, 2007). "Stress-dependent seismic anisotropy of shales" by Sayers (GEOPHYSICS, 1999). "Elastic piezosensitivity of porous and fractured rocks" by Shapiro (GEOPHYSICS, 2003). "Porosity and elastic anisotropy of rocks under tectonic stress and pore-pressure changes" by Shapiro and Kaselow (GEOPHYSICS, 2005). "The nanogranular nature of shale" by Ulm and Abousleiman (Acta Geotechnica, 2006). "Elastic properties of dry clay mineral aggregates, suspensions and sandstones" by Vanorio et al. (Geophysical Journal International, 2003). TLE

Acknowledgments: The experimental work was performed under the aegis of the IPETS Consortium cofunded by Chevron, Woodside, Anadarko, Origin, Santos, Schlumberger, and PIRSA. Permission to publish from these sponsors is much appreciated. Statoil is thanked for supplying the North Sea shale. The Geological Survey of Western Australia are thanked for supplying the Officer Basin shale. We are also grateful to sponsors Chevron and Origin for the Carnarvon and Bass Basin shales. Boris Gurevich and Utpalendu Kuila were formerly with CSIRO Petroleum, Perth, Australia.

Corresponding author: Marina.Pervukhina@csiro.au 